\title{
Understanding and Controlling E-beam Damage in Operando EC-STEM
}

B. Layla Mehdi ${ }^{1}$, Weiqun $\mathrm{Li}^{1}$, Juhan Lee ${ }^{1}$, Daniel Nicholls ${ }^{1}$, Nigel Browning ${ }^{2}$ and Andrew Stevens ${ }^{2}$

${ }^{1}$ University of Liverpool, Liverpool, England, United Kingdom, ${ }^{2}$ Sivananthan Laboratories, Inc., Bolingbrook, Illinois, United States

The demand to extend the driving range of electric vehicles has created a need for improved performance of Li-ion batteries, with both a higher energy density and a high-power capability being desirable. Accomplishing this requires the development and most importantly, the integration of new battery nanomaterials with high stability electrolytes. Here, high spatial resolution Operando experimental techniques can provide the key insights necessary to investigate the dynamic structural changes and processes that occur locally at the electrode/electrolyte interface during battery operation. The sheer complexity of the competing electrochemical processes that occur at the electrode/electrolyte interface during the battery cycling requires careful calibration of the system prior to operando observations to circumvent the effects of the electron beam. These effects include the formation of a wide range of radiolytic species, a $\mathrm{pH}$ change as well as various degradation mechanisms in organic, Li-based electrolytes which have yet to be fully understood. Here, we focus on understanding the stability of electrolyte [1] as a function of cycling performance, possible side reactions, stability of Li -anode and controlled formation of a solid-electrolyte interphase (SEI) layer. The process of the SEI layer formation continuously consumes the electrolyte components creating highly resistive layer, which leads to the rapid decrease of cycling performance and degradation of the Li anode. The growth of Li metal dendrites contributes to rapid capacity fading, which leads to an increased overpotential and, in the case of continuous growth an internal short circuiting of the battery creating extreme safety issues [2]. To obtain "dendrite free" cycling we investigate the role of additives [3] and well as possible cell contamination from unwanted side reactions both on cathode and anode. In order to minimise the electrolyte beam damage and achieve a faster imaging speed, we employ a sub-sampling and inpainting approach and link this result to our previous work under typical EM operating conditions for LiPF6 in PC electrolyte under $0.3 \mathrm{e} / \mathrm{A}^{2} \mathrm{~s}$ dose rate [2]. We use Ag nanoparticles (NP) as a calibration standard (Figure 1) [4] to understand the deviations in nucleation and growth under sparse sampling conditions compared to the conventional line scan in the scanning transmission electron microscope (STEM). In particular, we use this analysis to define an optimum probe separation that generates the maximum level of useful image information for the minimum amount of dose, dose rate and dose overlap for each experiment. In this presentation we will discuss the practical aspects of implementing sparse sampling and inpainting to perform precise dose-controlled experiments for a wide range of energy storage materials and processes [5]. 


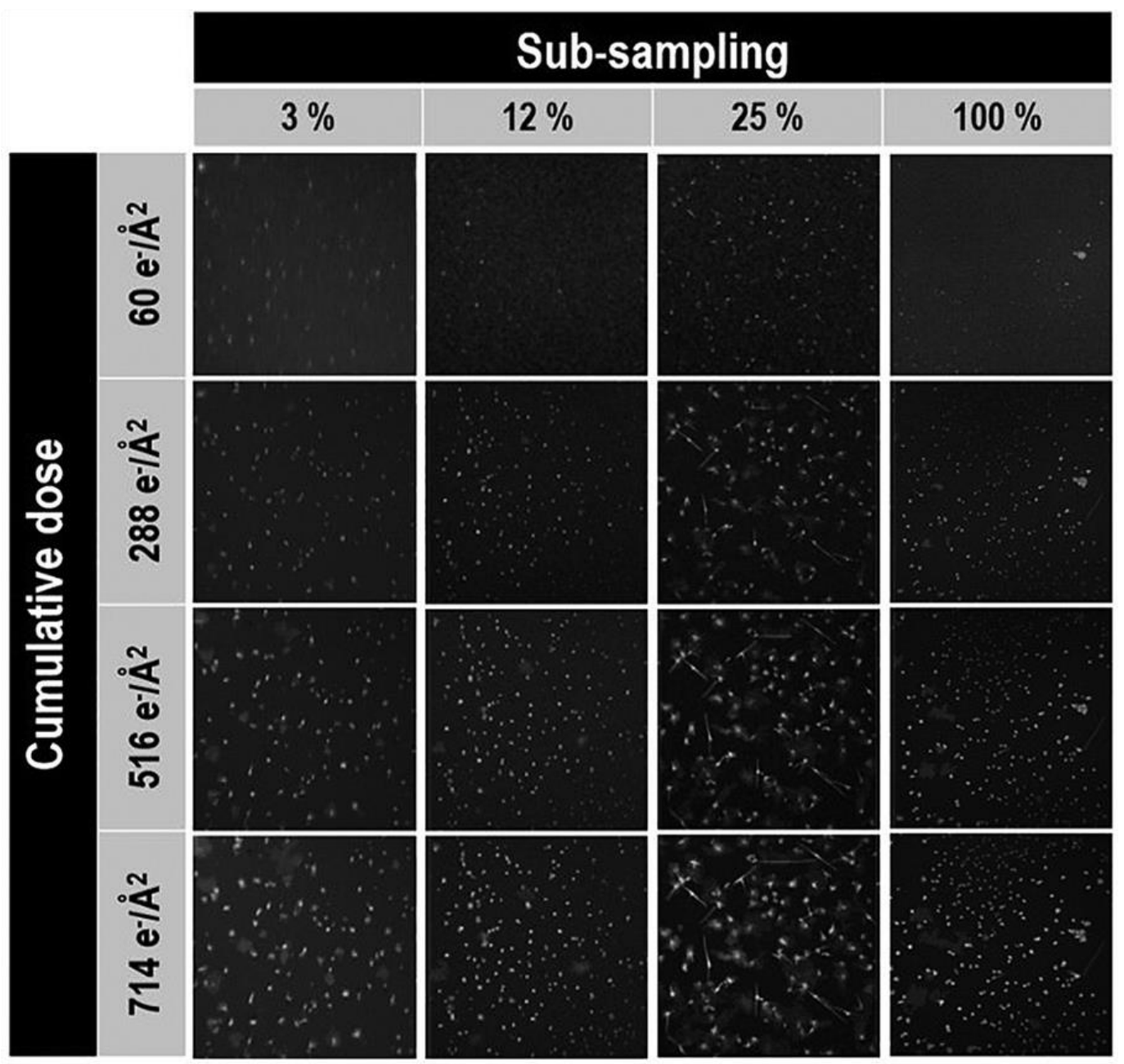

Figure 1. A set of subsampled images taken from different cumulative doses (i.e., time stamps) in the insitu nucleation and growth of Ag nanoparticles. For each of the images, the cumulative dose is controlled by the magnification, scan speed, and subsampling of the images. [4]

\section{References}

1. P. Abellán, C. Park, B. L. Mehdi, W. Xu, Y. Zhang, L. R. Parent, M. Gu, I. Arslan, J. Zhang, C. M. Wang, J. E. Evans, N. D. Browning, Nano Letters 14, 1293 (2014)

2. B. L. Mehdi, E. Nasybulin, J. Qian, C. Park, D. A. Welch, R. Faller, H. Mehta, W. A. Henderson, W. Xu, C. M.Wang, J. E. Evans, J. -G. Zhang, K. T. Mueller, and N. D. Browning, Nano Letters 15, 2168 (2015)

3. B. L. Mehdi, A. Stevens, J. Qian, C. Park, W. Xu, W. A. Henderson, J.-G. Zhang, K. T. Mueller, N. D. Browning, Scientific Reports 6, 34267 (2016)

4. B. L. Mehdi, A. Stevens, L. Kovarik, N. Jiang, H. Mehta, A. Liyu, S. Reehl, B. Stanfill, L. Luzi, W. Hao, L. Bramer, N. D Browning, Applied Physical Letters 115, 6, 063102 (2019)

5. This work was supported by the U.K. Faraday Institution. A portion of the results were obtained using the Environmental Molecular Sciences Laboratory (EMSL), a national scientific user facility sponsored by the Department of Energy's Office of Biological and Environmental Research and located at PNNL. PNNL is a multi-program national laboratory operated by Battelle for the U.S. Department of Energy (DOE) under Contract DE-AC05-76RL01830. 